\title{
The mechanism of atopic march may be the 'social' event of cells and molecules (Review)
}

\author{
JUNMING LUO ${ }^{1}$, YINGXIN $\mathrm{LI}^{2}$ and RUJUN GONG ${ }^{3}$ \\ ${ }^{1}$ Department of Pathology, Second Xiangya Hospital, Central South University Xiangya School of Medicine, Changsha, \\ Hunan, P.R. China; ${ }^{2}$ Eshelman School of Pharmacy, University of North Carolina, Chapel Hill, NC, USA; \\ ${ }^{3}$ Department of Medicine, Brown Medical School, Providence, RI, USA
}

Received July 7, 2010; Accepted August 9, 2010

DOI: 10.3892/ijmm_00000525

\begin{abstract}
The skin, the conjunctivae, the airways and the digestive tract compose a huge vulnerable biological surface, which is exposed to the external environment. An allergen can often trigger an allergic reaction at a number of sites or result in an atopic march. However, the mechanism of atopic march remains unclear. Less attention has been paid to the connection between the primary site and the atopic site, because current knowledge is established directly against harmful factors. Allergic hypersensitivity manifests in parts of the human body far away from the allergen. Growing evidence suggests that the epithelial cells serve as the 'engine' which initiates an allergic reaction through the production of large quantities of cytokines, chemokines and growth factors. Because the epithelial cells cover the entire surface of the skin, the conjunctivae, the airways, and the digestive tract, and are positioned at the terminals of neurons and the blood supply, the connection between the primary site and the atopic site can not be easily understood by the current knowledge of anatomy and of the neuroendocrine immune network. What is the linkage between these huge vulnerable biologic surfaces? This article highlights selected frontiers in allergy research of atopic march, and focuses on recently attained insights into the cellular and molecular events of primary and atopic lesions in the allergy progress. Special attention is paid to the homogeneity of the cellular and molecular events on the huge vulnerable surface. Based on currently available data we conclude that the skin, conjunctivae, airways and digestive tract may join together to form the frontier 'commonwealth union' in order to fight the allergen. The epithelial cells are the 'engine' as well as the main target which initiates both primary and atopic inflammatory reactions. The atopic lesion
\end{abstract}

Correspondence to: Dr Junming Luo, Department of Pathology, Second Xiangya Hospital, Central South University Xiangya School of Medicine, 156 Renmin Road, Changsha, Hunan 410011, P.R. China

E-mail: jluo099@yahoo.com

Dr Rujun Gong, Department of Medicine, Rhode Island Hospital, Brown Medical School, 593 Eddy Street, Providence, RI 02903, USA E-mail: rgong@brown.edu

Key words: allergy, atopic march, social event, epithelia, surface may 'duplicate' the primary contacted site of cellular and molecular events. The atopic march may be due to the intrinsic 'social' involvements of the positioned epithelial cells, but may not be totally controlled by the anatomic connection or the circulating systemic factors involved in allergy pathogenesis.

\section{Contents}

1. Introduction

2. The surface of the skin, the conjunctivae, the airways and the digestive tract may be a 'commonwealth union'

3. The epithelial cells are the initial and main target of the organ involved in the allergy pathogenesis

4. The 'social manner' of cells and molecules

5. Possible evidence of the 'social manner' of cells and molecules

\section{Introduction}

Allergic diseases affect approximately one-third of the population and constitute one of the major health care problem in the Western world $(1,2)$. Allergy is an inappropriate inflammatory reaction to an antigen. The allergen triggers an exaggerated immune response, and can aggravate an allergic response on the entire surface of the skin, the conjunctivae, the airways and the digestive tract (3-5). The entire surface of the skin, conjunctivae, airways and of the digestive tract has been activated to fight allergens, although most of them are not directly exposed to the harmful factor. The skin, conjunctivae, airways and the digestive tract may form a 'commonwealth union' to fight allergens anywhere on the huge vulnerable biologic surface. The communication among the surface 'commonwealth union' has been clinically documented during immediate allergic reactions or atopic march events. An inflammatory reaction is a protective response against the harmful factors; an atopic inflammatory reaction may indicate a close relationship between the primary site and atopic sites in the pathogenesis of an allergy (6-9). Although more and more information is accumulating about the molecular events mediating a series of cellular events such as activation, degeneration, apoptosis, necrosis, 
differentiation, hyperplasia, carcinogenesis and inflammation caused by the direct harmful factor exposure, there is still not enough knowledge to understand the intrinsically distal communication. It still remains unknown why in an allergic reaction there are homogeneous cellular and molecular events which take place not only at the primary-contacted site of the airways, skin or digestive tract but also to the not directly exposure distal sites, and there can even be a whole body response. What is the mechanism of the immediate atopic allergic reaction or atopic march? In this article, we we provide an overview of the cellular and molecular events with a special emphasis on the homogeneity between the primary contacted sites and the secondary 'passive' inflammatory response sites of the systemic allergic involvements. This phenomenon is not easily understood by current knowledge of anatomy and the neuroendocrine immune network. To the best of our knowledge, in the allergy pathogenesis, the homogeneous cellular and molecular inflammatory events take place at the directly-exposed sites and the not directly-exposed atopic sites. The allergenmediated homogeneous cellular and molecular events may be initiated by cytokines or chemokines produced by local epithelial cells, but may not be totally controlled by an anatomic connection or circulating systemic factor mediation. An allergen may trigger the 'social manner' of cells and molecules in allergy pathogenesis. The indirectlyexposed sites may 'duplicate' the 'social' inflammatory reaction of the directly-exposed site.

Our body is a highly ordered organ system. The communication among the organs such as the lung and the colon is a clinically-observable event (10-12). The organ is not a mixture of a bunch of cells and molecules. We hypothesize that each individual cell or molecule not only plays its local role in the positioned organ, but may also play a 'social' role on the distal communication during exposure to harmful factors $(11,13-14)$. In fact, the 'social property' of cells and molecules is a clinically-documented event $(13,14)$. The entire surface of the skin, the conjunctivae, the airways and the digestive tract may be intrinsically involved in a 'social' inflammatory reaction to fight allergens. In the allergic pathogenesis, although cells of the epithelium, mast cells, T helper 2 (Th2) lymphocytes and the molecules of immunoglobulin E (IgE), chemokines and cytokines are located in different parts of the body, they may 'socially' respond in the systemic involvements of allergy. The indirectly-exposed sites of the skin, the conjunctivae and of the respiratory, alimentary, or reproductive tracts and even of some solid organs may form a huge 'commonwealth union' which 'socially' responds and contributes to the pathogenesis of a distal allergic reaction.

\section{The surface of the skin, the conjunctivae, the airways and the digestive tract may be a 'commonwealth union'}

The skin, the conjunctivae, the airways, and the digestive tract have all been implicated in the systemic involvements of allergy. The entire surface of the skin, the conjunctivae, the airways, and the digestive tract may form a clinicallyobservable 'commonwealth union'.
Allergen sensitization and challenge in the skin and the systemic response. When a patient gets in contact with latex, an allergic reaction may occur immediately or develop later (15-19). The symptoms of the reaction include hives and urticaria. The skin reaction may occur anywhere in the body and not necessarily at the site where the direct contact with the latex occurred $(15,16)$. In the meanwhile, an allergic reaction may occur at the conjunctivae, airways and digestive tract even at the whole body. The symptoms of this allergic reaction include wheezing; coughing; shortness of breath; sneezing; nasal congestion; runny nose; conjunctivitis (red, itchy, watery eyes); nasal, palatal, or ocular itching; nasorhinitis (chronic runny nose); asthma; hypertension; and anaphylaxis, a serious and potentially life-threatening allergic response. The latex-triggered hypersensitivity reaction may affect the entire surface of the skin, the conjunctivae, the airways and the digestive tract (15-19). Obviously, there is a clinically-observable connection between the skin, and the conjunctivae, airways and the digestive tract. A latex allergy has often been associated wtih the presence of a concomitant food allergy (19).

Allergen sensitization and challenge in the digestive tract and the systemic response. Similarly, in food-mediated allergy, the food hapten may also trigger an exaggerated inflammatory reaction on all the members of this 'commonwealth union', and cause itching; scratchy throat; hives anywhere on the body; swelling of the eyelids, face, or tongue; nausea; cramps; vomiting; diarrhea; nasal congestion; shortness of breath or wheezing (20-24). When the blood pressure drops down, the airways are blocked, and the throat is closed, the reaction can be serious and potentially life-threatening and can eventually progress into anaphylaxis (20-24). Therefore, there is a clinically-observable connection between the digestive tract and the skin, conjunctivae, and airways.

Allergen sensitization and challenge in the airways and the systemic response. When a patient inhales pollen, pollinosis is not limited to rhinoconjunctivitis and/or bronchial asthma, but may intrinsically extend to an inflammation of the digestive tract and the skin (25-27). The symptoms of the reaction are very similar to latex contact and food allergy (27). The connection between the airways and the conjunctivae, the skin and the digestive tract is a clinically-observable event in the pathogenesis of pollinosis.

Latex allergen, food protein and the airways source of pollen and house mites, all may aggravate an inflammatory reaction on entire surface of the skin, conjunctivae, airways and of the digestive tract although most of them are not sites of direct hapten exposure. These symptoms of latex allergy can be exacerbated in certain people when specific foods, such as hevamine-containing fruits, are ingested (19). Food allergy and asthma commonly co-exist in the same patient $(20,27)$. Approximately one-third of children with a food allergy have asthma, and 4-8\% of children with asthma have food allergies (20). As mentioned before, allergic diseases typically develop on the entire mucosal surface of this 'commonwealth union'. The allergic inflammatory reaction site may not be in direct contact with the allergen. The directly-contacted allergen site can drive an allergic inflam- 


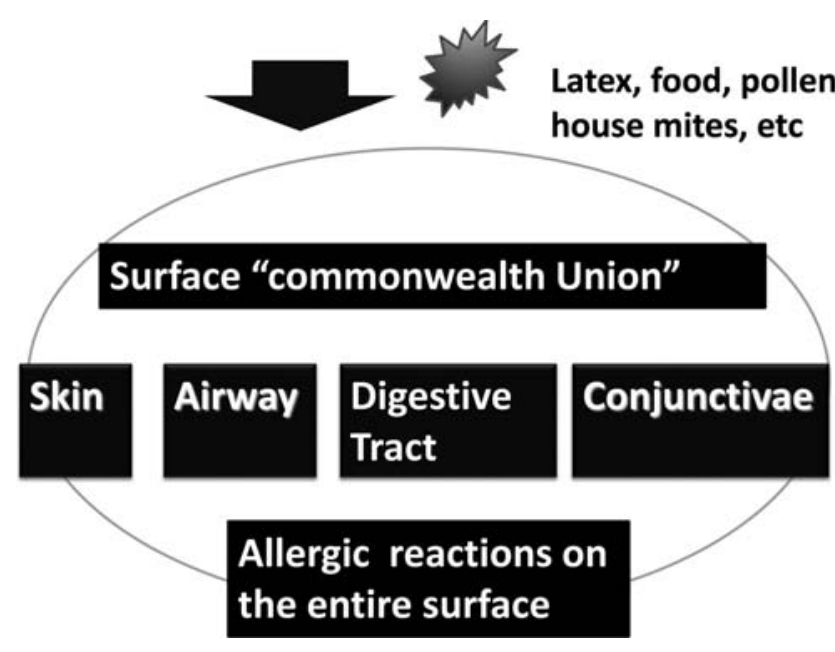

Figure 1. Model for allergens triggering the entire surface of allergic reactions. Allergens such as latex, food hapten, pollen or house mites may contact any site(s) on the surface of the skin, the conjunctivae, the airways and of the digestive tract, aggravating the entire surface and resulting in an immediate allergic reaction or a late-developed atopic march. The skin, conjunctivae, airways and digestive tract join a huge 'commonwealth union' to fight allergens. Allergic hypersensitivity manifests in parts of the body which are not in direct contact with the allergens.

mation anywhere on the surface of this 'commonwealth union'. The allergen can elicit an inflammatory reaction on all the members of the 'commonwealth union' regardless of the primary-contacted site (Fig. 1).

\section{The epithelial cells are the initial and main target of the organ involved in the allergy pathogenesis}

The entire surface of the skin, conjunctivae, airways and digestive tract forms a clinically-observable 'commonwealth union' during the systemic involvement of allergy. The epithelial cells cover the entire mucosal surface of the 'commonwealth union'. More and more evidence is accumulating that the epithelial cells could be considered not only the frontier sentinels and barriers, but also the central participants in the innate and adaptive immune responses as well as in mucosal inflammation. The epithelial cells can release large quantities of proinflammatory cytokines, growth factors and chemokines that attract inflammatory cells to initiate and sustain an inflammatory reaction (28-32). The epithelial cells of the entire surface of the skin, the conjunctivae, the airways and the digestive tract are also the main targets during the pathogenesis of the systemic involvement of allergy (Fig. 2).

The epidermal keratinocyte is the initial and main target of the allergic skin involvement. The skin, which covers the entire body, is continuously exposed to the external environment. It serves as a protective barrier: microorganisms find it almost impossible to traverse the epidermis, and water loss is inhibited. The skin has the potential to provide a window into the patient and aid in the diagnosis of diseases of all organ systems. Disorders of every organ may cause skin symptoms and signs (33). Physicians of Traditional Chinese Medicine (TCM) believe that skin is a 'Biao' (surface) which always relates to disorders of the 'Li's' (internal organs). The skin may be the favorable and easiest observable organ for studying

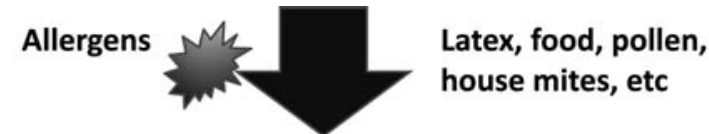

Epithelial cells anywhere on the skin,
conjunctivae, airways and digestive tract

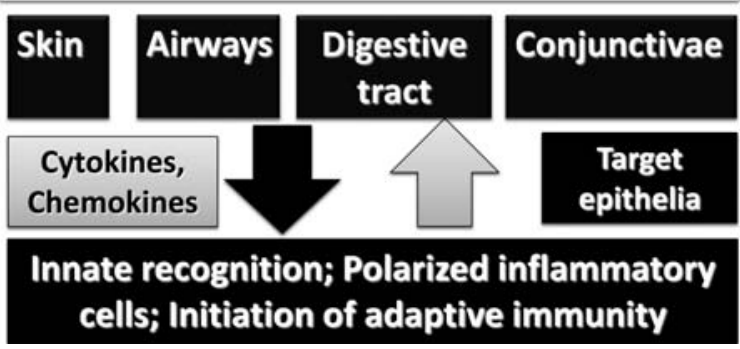

Figure 2. Model summarizing the epithelial cells as an 'engine' to initiate an allergic reaction and main target in allergy pathogenesis. The epithelial cells cover the surface of the skin, conjunctivae, airways and digestive tract, and are the first in line to fight allergens. The epithelial cells are the central participants of the innate recognition, polarization of inflammatory cells, and initiation of adaptive immunity through cytokines and chemokines. On the other hand, the mesenchymal inflammatory cells target the epithelial cells.

the pathogenesis of the systemic involvement of allergy, because during the allergic progress it always elicits an inflammatory reaction even though the primary allergen contact site is located in the skin, airways or digestive tract.

Allergic skin disorders include urticaria, angioedema, contact dermatitis and atopic dermatitis (AD) (34). Contact dermatitis is a localized rash or irritation of the skin caused by contact with a foreign substance. Inflammation of the affected tissue is present in the epidermis and dermis where direct contact with the hapten takes place (34). Urticaria, angioedema, and $\mathrm{AD}$ are atopic disorders, in which contact of a specific allergen with the surface of the gastrointestinal or the respiratory tract drives an allergic reaction from the primary allergen contacted sites to the skin $(30,34)$. Urticaria and angioedema are wheals, characterized by central swelling surrounded by erythema, associated with itching. In the inflammatory process, both the dermis and the subcutis are involved, but the main target is the epidermis $(30,34)$. AD is characterized by eczematous changes in the epidermis, and most patients have an atopic background with past or family histories of bronchial asthma, allergic rhinitis, and/or allergic conjunctivitis. Infiltration of lymphoid cells into the epidermis and upper dermis can be observed under the microscope (34). The epidermal keratinocytes, which are triggers of immune abnormalities, as well as peripheral effectors, are important to further disclose the pathogenesis of this enigmatic disorder $(29,30,34)$. Although the involved layer is different in individual cases of allergic skin diseases, the keratinocytes which produce cytokines and chemokines have been considered as the crucial factors in the initiation of skin inflammation $(29,30,24)$. Therefore, the epidermal keratinocytes are believed to be the 'engine' initiating skin inflammation. The epidermal keratinocytes are also believed to be the main target of eczema and the key participants in the allergic skin disorders $(29,30)$. Because the epidermal keratinocytes are positioned at the first line to fight harmful factors, it would be easy to postulate that allergens drive these cells to release 
cytokines and chemokines and to polarize the inflammatory cells under exposure. If there are no allergens contacting the surface of the skin, why are the epidermal keratinocytes still producing cytokines and chemokines and polarizing the inflammatory cells? There should be a communication among the epidermal keratinocytes and the airway epithelial cells, digestive tract and conjunctivae. In fact, a latexmediated allergic reaction may occur anywhere in the body and not necessarily at the site where direct contact with the latex occurred. So there also should be a communication between the epidermal keratinocytes of the latex-contacted site and the epidermal keratinocytes of the site not directly contacted by latex.

The airway epithelium is the initial main target in allergy. The airway epithelial cells, which are at the first line of exposure to many pathogens, serve as a complex physical barrier that defends against exposures to potentially harmful inhaled substances and microbial pathogens. A breakthrough in the understanding of the ability of the innate immune system to rapidly recognize pathogens occurred with the discovery of the Toll-like receptors (TLRs). TLRs were originally identified as homologues of Drosophila Toll. To date, 10 human TLRs have been identified (35-37). Airway epithelial cells express mainly TLR2-6, which plays a crucial role on the innate immune recognition $(28,32,38,39)$. TLR signaling can lead to activation of several transcription factors such as NF- $\mathrm{BB}$, and interferon (IFN) regulatory factors (IRF) 3, 5 and 7. The activation of NF- $\mathrm{\kappa B}$ induces proinflammatory genes such as TNF, interleukin (IL)-6 and IL-12 expression (28).

Activated airway epithelial cells can induce the synthesis and secretion of immune cell-mediated host defense molecules $(28,40)$, which result in an immediate host defense. Host defense molecules such as antimicrobial and antiviral proteins belong to proinflammatory cytokines which can activate other mucosal innate immune cells. The major antimicrobial products secreted constitutively or inducibly by epithelial cells include lysozyme, lactoferrin, defensins, collectins, pentraxins, LL-37, the secretory leukocyte protease inhibitor and serum amyloid A (40). In the allergic inflammatory reaction, the activation of Th2 cells and secretion of IL-4, IL-5, and IL-13 is linked with an impaired antibacterial response (28).

Activated innate immune responses can secondarily induce the recruitment and activation of dendritic cells (DCs) that amplify antigen recognition, antibody production and other adaptive immunity $(28,32,38-40)$. Epithelial DCs are recruited from the blood. Airway epithelial cells are capable of inducing migration of DCs into the epithelium via CCL20 (MIP-3 $\alpha$ ) production (41). Epithelial cells in collaboration with lymphocytes contribute to the recruitment and the differentiation of DCs from circulating monocytes. The process of attraction and differentiation of monocytes is driven by pulmonary epithelial cells, Th2 cells, natural killer (NK) cells or basophils (28). The GM-CSF and IL-15 from the pulmonary epithelial cells are important factors in this process (28). Epithelial cells may enhance an allergic airway inflammation by inducing DC-mediated Th2 differentiation and also increase mast cell activation via the production of thymic stromal lymphopoietin (TSLP) $(9,28,32)$.
Activated innate immune responses can also secondarily induce recruitment and activation of $\mathrm{T}$, and $\mathrm{B}$ lymphocytes that amplify antigen recognition, antibody production and other adaptive immunity $(9,28,32,38-40)$. Local T lymphocyte responses and immunoglobulin production are quite important both for the protection from pathogens and in the pathogenesis of various types of inflammatory diseases of the airways $(9,32)$. Therefore, the epithelial cells are the central participants in the regulation of both the innate and the adaptive immune responses in airway inflammation (32). Most of asthma patients exhibit a Th2 inflammatory response with coordinated up-regulation of cytokines produced by epithelial cells; however, over-interpretation of the immunologic pathway has led to a simplistic view that asthma results purely from allergen sensitization and exposure (32). Allergens can break tissue homeostasis and dysregulate the local tissue microenvironment. The aberrant epithelial injury/repairs were believed as the mechanism of allergic inflammation of the airways $(9,28,32,38-40)$.

Skin contacted latex allergen, food protein, and the airways source of pollen and house mites, all can exacerbate the whole airway inflammatory reaction. Bronchial asthma is a common chronic disorder of the airways characterized by bronchial hyperresponsiveness, airflow obstruction and an underlying inflammation which affects about $7 \%$ of the population in the United States (20). Bronchial asthma frequently accompanies $\mathrm{AD}$, allergic rhinitis, and allergic conjunctivitis (20). More and more evidence is accumulating that both the skin epidermal keratinocytes and the airway epithelial cells are the initial main target of the inflammatory reaction (9). Less attention has been paid to the epithelial cells of the digestive tract. These cells may behave like the epidermal keratinocytes of the skin and the airway epithelial cells. The epithelial cells produce cytokines and chemokines, and drive the inflammatory cell polarization. Viewing asthma primarily as an epithelial disease with adoption of a chronic wound scenario also provides a route to the airways wall remodeling and asthma phenotypes varying over the course of life (32). The mechanism should be either the systemic, factor-mediated cellular and molecular events or the intrinsic connection between the primary and the atopic sites. As is known, the epithelial cells are located at the terminals of the neurons and the blood supply. Therefore, the systemic factormediated connection is not easy to be totally understood by the current knowledge of anatomy, and of the neuroendocrine immune network. These cellular and molecular events may relate to the intrinsic 'social manner' of cells and molecules.

\section{The 'social manner' of cells and molecules}

In the allergy pathogenesis, the surface of the skin, conjunctivae, airways, and digestive tract has been involved in the inflammatory reaction to fight allergens. They may form a huge 'commonwealth union' against the allergen. What are the cellular and molecular events taking place in the huge 'commonwealth union'?

The linkage of epithelial cells in different sites may be through an intrinsic 'social' involvement and not by systemic factor mediation. Atopic diseases include eczema (AD), asthma, 


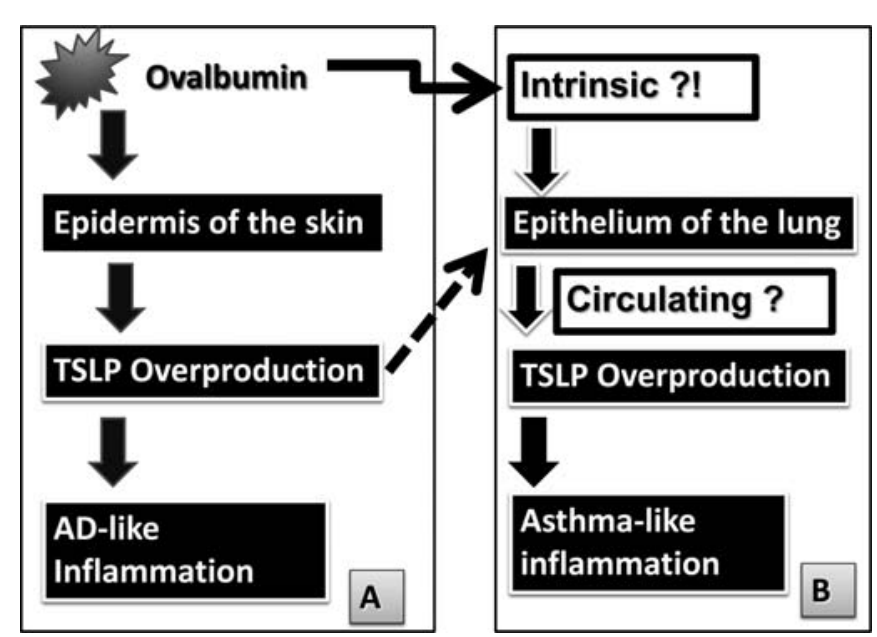

Figure 3. Model summarizing how ovalbumin drives the skin and lung into inflammation. This figure shows ovalbumin driving both the skin and the lung into inflammation. Part A shows atopic dermatitis-like lesion of the skin, in which TSLP over-production by the epidermal keratinocytes underlies the ovalbumin sensitization and challenge. Part B shows an asthmalike lesion of lung, in which epithelial cells overproduce TSLP underlying the ovalbumin sensitization and challenge. The ovalbumin sensitization and challenge are crucial factors driving both the skin and lung into inflammation. The relationship between part A and part B may be through an intrinsic 'social' connection of the epidermal keratinocytes of the skin and the epithelial cells of the lung, but may not be through the circulating TSLP from epidermal keratinocytes targeting epithelial cells of the lung.

allergic rhinitis, etc. AD is often the initial step in the atopic march; up to $70 \%$ of patients with severe AD develop asthma later in life $(7,42,43)$. AD and asthma share an atopic phenotype that includes Th2 inflammation with eosinophilia and hyper-IgE immunoglobulinemia. However, the molecular mechanisms underlying the atopic march remain unclear $(7,9,32,42,43)$. More and more evidence has been accumulating that the epithelial cells are the key participants, which initiate allergic inflammation, as well as the main targets in the pathogenesis of allergy. Activated epithelial cells may produce large quantities of proinflammatory cytokines and chemokines which drive inflammatory cell polarization, and elicit inflammation. Recently, TSLP, a general biomarker for skin-barrier defects $(7,9,32)$ and an IL-7-like cytokine produced by epithelial cells, emerged as a potential master regulator of both skin and the airways inflammation (7,9,32). TSLP signaling plays an important role in the allergic airway and skin inflammation. Zhang et al (9) found that increased TSLP expression in skin keratinocytes not only locally triggered $\mathrm{AD}$-like lesions in the skin of mice, but also lead to an aggravation of a concomitant ovalbumin (OVA)-induced asthma-like lung inflammation (Fig. 3). Furthermore, Zhang et al elucidated that an increased TSLP expression in epidermal keratinocytes and the subsequent increase in blood circulating TSLP in their mouse model did not lead to spontaneous lung inflammation in the absence of OVA sensitization and challenge (9). In fact, there are high TSLP mRNA expression levels in bronchial epithelial cells and in the submucosa of asthmatic patients (44), which indicate that TSLP locally produced in the lung could be important in the development and/or maintenance of asthma. We may conclude that TSLP is a crucial factor driving both the skin and the lung into inflammation. However, an increased TSLP expression in epidermal keratinocytes and a subsequent increase in blood circulating TSLP are not enough to drive both the skin and the lung into inflammation (9). Obviously, the lung inflammatory reaction is not triggered by circulating TSLP, and it may be produced by the pulmonary epithelial cells themselves. Since the epithelium is on the terminal of the blood supply, an atopic allergic response, especially an immediate allergic response is not easy to be understood by current knowledge of anatomy. OVA sensitization and challenge are extremely important to drive both the skin and the lung into inflammation. The intrinsic connection between the skin and the lung may be through locally produced TSLP underlying OVA sensitization and challenge. This intrinsic communication among heterogeneous epidermal keratinocytes and airway epithelial cells may relate to the 'social manner' of epithelial cells. Allergens, such as OVA, trigger this potential 'social property' of the epithelial cells (Fig. 3).

The 'social manner' of cells and molecules in allergy pathogenesis. In the allergy pathogenesis, allergens such as OVA drive both the skin and the lung into inflammation. Allergens are a crucial factor connecting skin epidermal keratinocytes and lung epithelial cells. Cytokines and chemokines, such as TSLP, released from activated epithelial cells break the local microenvironment and the tissue homeostasis of the epithelial-mesenchymal trophic unit and trigger the inflammatory reaction $(32,45)$. The epithelial cell-mediated inflammatory cellular and molecular events have been extensively studied and well established. The inflammatory reaction occuring at the huge vulnerable biological surface is a bunch of homogeneous cellular and molecular events. Mast cells are key cells in the pathogenesis of IgE-dependent hypersensitivity reactions (46). Mast cells are connective tissue cells which are widely distributed throughout the body predominantly near blood vessels and nerves (46). Evidence is accumulating that allergens trigger mast cell activation through the epithelial cells $(30,46)$. It is very difficult to understand the mechanism of the mast cell-mediated allergic reaction, especially the immediate atopic allergic reaction through the systemic factors from the blood or nerves. Mast cells and IgE molecules may be intrinsically activated through allergen-mediated epithelial cells. Both bronchial asthma and $\mathrm{AD}$ are characterized by Th2-mediated chronic inflammatory diseases in response to allergen $(7,9,32,34)$. Locally-produced but not circulating TSLP has been postulated to be a master regulator of Th2 inflammation with eosinophilia and hyper-IgE immunoglobulinemia (9). In accordance, most of atopic cellular and molecular events are induced by the locally-produced initial regulators and not by circulating regulators. The majority of locally produced initial regulators come from allergen-activated epithelial cells $(9,32)$. An allergic disorder may be an epithelial disease with adoption of a wound scenario (32). The manner in which circulating regulators directly contact the allergen may relate to the 'social property' of cells and molecules. Therefore, the homogeneous cellular and molecular events of an immediate atopic allergic reaction and/or the atopic march may relate to the dysfunction of the 'social manner' of cells and molecules. 


\section{Possible evidence of the 'social manner' of cells and molecules}

The skin, conjunctivae, airways, digestive and reproductive tract is a huge vulnerable biological surface, which is continuously exposed to the environment. The epithelial cells cover the entire surface, and are the first line to fight any possible harmful factors. The epithelial cells play a crucial role in the innate immune recognition, the host defense. The direct or indirect interaction of epithelial cells with mast cells, T, and B lymphocytes, DCs, eosinophils, neutrophils, and basophils has been extensively studied and well documented (28). The epithelial cells on the entire surface form a 'commonwealth union' and intrinsically respond to harmful factors such as allergens. The 'social manner' of cells and molecules is a useful paradigm to describe the intrinsically distal communication.

Filaggrin and atopic march. Filaggrin as a major predisposing gene for atopic disease has caused a major paradigm shift in dermatology and allergy research (47). Filaggrin is a skin barrier protein in the granular layer of the epidermis and aggregates keratin filaments in the cell to form the cornified envelope, which is critical for maintaining an effective skin barrier against the environment $(20,47,48)$. Recent human genetic studies strongly suggest that perturbation of the skin barrier function as a result of reduction or complete loss of filaggrin expression leads to enhanced percutaneous transfer of an allergen (47-49). The expression level of filaggrin has been known to be decreased in AD patients at both the protein and mRNA levels $(50,51)$. The filaggrin gene defect may be the foundational predisposing factor not only for the development of eczema, but also for the initial sensitization and progression of the allergic diseases $(47,48)$. AD patients with filaggrin loss-of-function mutation exhibit an increased incidence of asthma (52). In fact, filaggrin gene expression is limited to the skin and the oral mucosa and is absent in the epithelial cells of the upper and lower airways, and digestive tract $(47,53)$. The loss-of-function mutation of the filaggrin gene is not only directly involved in dysfunction of the skin barrier, but is also indirectly involved in barrier dysfunction of the upper and lower airways and of the digestive tract (54). The mechanism by which the dysfunction of the skin barrier leads to the atopic march of the airways and the digestive tract remains unknown. The atopic march underlying the loss-of-function mutation of filaggrin may break the 'social' connection of the 'commonwealth union'.

In conclusion, allergic hypersensitivity manifests in parts of the human body which are not in direct contact with the allergen. Allergic reaction is an epithelial cell-mediated inflammation. The connection between the skin, conjunctivae, airways and the digestive tract may relate to the 'social manner' of the cells and molecules.

In human beings, the organs, cells and molecules are 'social' members of the body $(11,13,14)$. This is a plausible hypothesis. For example, a latex-mediated allergic reaction may occur anywhere in the body or the skin and not necessarily at the site of direct contact with latex. How can current knowledge of anatomy, and neuroendocrine immunity provide evidence of the connection between the latex contacted site and the non-contacted site? The concept of the 'social manner' of cells and molecules would be a plausible concept to understand the atopic march and to gain knowledge on the cellular and molecular mechanisms of TCM. The physicians of TCM believe that the body is an open system. The TCM physician will first carefully examine the patients' skin; tongue and conjunctivae then proceed to further examination and make a diagnosis. The skin, tongue and conjunctivae are believed as a 'Biao' (surface) which always relates to disorders of the 'Li's' (internal organs). In the allergy pathogenesis, the allergen as a 'social' factor drives the epithelial cells of the skin, conjunctivae, airways and digestive tract into inflammation. The 'social manner' of cells and molecules could also help to attain insight into the pathogenesis of enigmatic complications such as inflammatory bowel diseases (IBD). For example, patients with chronic IBD (chronic ulcerative colitis and Crohn's disease) may have a variety of extraintestinal manifestations, including arthritis, ankylosing spondylitis, erythema nodosum, pyoderma gangrenosum, dermatitis, aphthous stomatitis, conjunctivitis, episcleritis, uveitis, hepatitis, pericholangitis, sclerosing cholangitis, primary biliary cirrhosis, pancreatitis, thyroiditis, pyelitis, and pericarditis (10-12). There are homogeneous inflammatory cellular and molecular events taking place in all of the involved organs. The complications of IBD may relate to a dysfunction of the 'social manner' of inflammatory cells and molecules due to the inflammatory cells and molecules present in all the involved organs.

\section{Acknowledgements}

We thank Dr Y. Eugene Chin and Dr Mukut Sharma for their critical reading of this manuscript.

\section{References}

1. Miyazaki D, Tominaga T, Yakura K, Kuo C-H, Komatsu N, Inoue $\mathrm{Y}$ and Ono SJ: Conjunctival mast cell as a mediator of eosinophilic response in ocular allergy. Mol Vis 14: 1525-1532, 2008.

2. Ono SJ: Molecular genetics of allergic diseases. Annu Rev Immunol 18: 347-366, 2000.

3. Yu LC: The epithelial gatekeeper against food allergy. Pediatr Neonatol 50: 247-254, 2009.

4. Zhang X, Li Y and Lian Y: Thinking and look forward to clinical study of allergic rhinitis by treatment of traditional Chinese medicine. Zhongguo Zhong Yao Za Zhi 34: 2419-2422, 2009.

5. Lin RY, Rodriguez-Baez G and Bhargave GA: Omalizumabassociated anaphylactic reactions reported between January 2007 and June 2008. Ann Allergy Asthma Immunol 103: 442-445, 2009.

6. Van Bever HP: Determinants in early life for asthma development. Allergy Asthma Clin Immunol 5: 6, 2009.

7. Demehri S, Mormoto M, Holtzman MJ and Kopan R: Skinderived TSLP triggers progression from epidermal-barrier defects to asthma. PLoS Biol 19: e1000067, 2009.

8. Curtiss FR: Atopic march to a dead end or does the theory really have legs? J Manag Care Pharm 13: 810-811, 2007.

9. Zhang Z, Hener P, Frossard N, Kato S, Metzger D, Li M and Chambon P: Thymic stromal lymphopoietin overproduced by keratinocytes in mouse skin aggravates experimental asthma. Proc Natl Acad Sci USA 106: 1536-1541, 2009.

10. Kirsner JB: The local and systemic complications of inflammatory bowel disease. JAMA 242: 1177-1183, 1979.

11. Luo J, Liu Z and Eugene CY: Overexpression of pulmonary surfactant protein A like molecules in inflammatory bowel disease tissues. Zhong Nan Da Xue Xue Bao Yi Xue Ban 33: 979-986, 2008. 
12. Casey MB, Tazelaar HD, Myers JL, Hunninghake GW, Kakar S, Kalra SX, Ashton R and Colby TV: Noninfectious lung pathology in patient with Crohn's disease. Am J Surg Pathol 27: 213-219, 2003.

13. Luo JM, Wan YS, Liu ZQ, Wang GR, Floros J and Zhou HH: Regularity of distribution of immunoreactive pulmonary surfactant protein A in rat tissues. Int J Mol Med 14: 343-351, 2004.

14. Luo JM and Wan YS: Tightly regulated distribution of family members of proteins is related to social property in the open body system. Int J Mol Med 17: 411-418, 2006.

15. Nienhaus A, Kromark K, Raulf-Heimsoth M, Kampen VV and Merget R: Outcome of occupational latex allergy-work ability and quality of life. PLoS One 3: e3459, 2008.

16. Binkley HM, Schroyer T and Catalfano J: Latex allergies: A review of recognition, evaluation, management, prevention, education, and alternative product use. J Athletic Train 38: 133-140, 2003.

17. Jaeger D, Kleinhans D, Czuppon AB and Baur X: Latexspecific proteins causing immediate-type cutaneous, nasal, bronchial, and systemic reaction. J Allergy Clin Immunol 89: 759-768, 1992

18. Noorani S, Richter A, Fenn M, Read G, Huissoon A, Williams P, William A and Krishna MT: Immediate hypersensitivity to latex in the absence of demonstrable specific immunoglobulin E. J Investig Allergol Clin Immunol 19: 419-420, 2009.

19. Brehler R, Theissen U, Mohr C and Luger T: 'Latex-fruit syndrome': frequency of cross-reacting $\operatorname{IgE}$ antibodies. Allergy 52: 404-410, 1997

20. Sicherer SH and Sampson HA: Food allergy. J Allergy Clin Immunol 117: S470-S475, 2006.

21. Bird JA and Burks AW: Food allergy and asthma. Prim Care Respir J 18: 258-265, 2009.

22. Eckman J, Saini SS and Hamilton RG: Diagnostic evaluation of food-related allergic disease. Allergy Asthma Clin Immunol 5: 2, 2009 .

23. Hodge L, Swain A and Faulkner-Hogg K: Food allergy and intolerance. Aust Fam Physician 38: 705-707, 2009.

24. Sicherer SH and Sampson HA: Food allergy: Recent advances in pathophysiology and treatment. Annu Rev Med 60: 261-277, 2009

25. Bartra J, Sastre J, del Cuvillo A, Montoro J, Jauregui I, Davila I, Ferrer M, Mollol J and Valero A: From pollinosis to digestive allergy. J Investig Allergol Clin Immunol 19 (Suppl 1): 3-10, 2009.

26. Simons FE: Anaphylaxis. Allergy Clin Immunol 121: S402-S407, 2008.

27. Yagami T: Allergies to cross-reactive plant protein. Latex-fruit syndrome is comparable with pollen-food allergy syndrome. Intl Arch Allergy Immunol 128: 271-279, 2002.

28. Kato A and Schleimer RP: Beyond inflammation: airway epithelial cells are at the interface of innate and adaptive immunity. Curr Opin Immunol 19: 711-720, 2007.

29. Terui T: Analysis of the mechanism for the development of allergic skin inflammation and the application for its treatment: overview of the pathophysiology of atopic dermatitis. J Pharmacol Sci 110: 232-236, 2009

30. Komine M: Analysis of the mechanism for the development of allergic skin inflammation and the application for its treatment keratinocytes in atopic dermatitis-their pathogenic involvement. J Pharmacol Sci 110: 260-264, 2009.

31. Xu W, He B, Chiu A, Chadburn A, Shan M, Buldys M, Ding A, Knowles DM, Santini PA and Cerutti A: Epithelial cells trigger frontline immunoglobulin class switching through a pathway regulated by the inhibitor SLPI. Nat Immunol 8: 294-303, 2007.

32. Holgate ST, Roberts G, Arshad SS, Howarth PH and Davies DE: The role of the airway epithelium and its interaction with environmental factors in asthma pathogenesis. Proc Am Thorac Soc 6: 655-659, 2009.

33. Lee A: Skin manifestations of systemic disease. Austr Fam Physician 38: 498-505, 2009.
34. Incorvaia C, Frati F, Verna N, D'Alo S, Motolese A and Pucci S: Allergy and skin. Clin Exp Immunol 153 (Suppl 1): 27-29, 2008.

35. Akira S, Uematsu S and Takeuchi O: Pathogen recognition and innate immunity. Cell 124: 783-801, 2006.

36. Lee MS and Kim YJ: Signaling pathways downstream of pattern-recognition receptors and their cross talk. Annu Rev Biochem 76: 447-480, 2007.

37. Trinchieri G and Sher A: Cooperation of Toll-like receptor signals in innate immune defense. Nat Rev Immunol 7: 179-190, 2007.

38. Sha Q, Truong-Tran AQ, Plitt JR, Beck LA and Schleimer RP: Activation of airway epithelial cells by toll-like receptor agonists. Am J Respir Cell Mol Biol 31: 358-364, 2004.

39. Homma T, Kato A, Hashimoto N, Batchelor J, Yoshikawa M, Imai S, Wakigushi H, Saito H and Matsumoto K: Corticosteroid and cytokines synergistically enhance toll-like receptors 2 expression in respiratory epithelial cells. Am J Respir Cell Mol Biol 31: 463-469, 2004

40. Schleimer RP, Lane AP and Kim J: Innate and acquired immunity and epithelial cell function in chronic rhinosinusitis. Clin Allergy Immunol 20: 51-78, 2007

41. Upham JW and Stick SM: Interactions between airway epithelial cells and dendritic cells: implication for the regulation airway inflammation. Curr Drug Targets 7: 541-545, 2006.

42. Ziegler SF and Artis D: Sensing the outside world: TSLP regulates barrier immunity. Nat Immunol 11: 289-293, 2010.

43. Spergel JM and Paller AS: Atopic dermatitis and atopic march. J Allergy Clin Immunol 112: S118-S127, 2003.

44. Ying S, O'Connor B, Ratoff J, Meng Q, Mallett K, Cousins D, Robinson D, Zhang G, Zhao J, Lee TH and Corrigan C: Thymic stromal lymphopoietin expression is increased in asthmatic airways and correlates with expression of Th2-attracting chemokines and disease severity. J Immunol 174: 8183-8190, 2005.

45. Holgate ST: Asthma: more than an inflammatory disease. Curr Opin Allergy Clin Immunol 2: 27-29, 2002.

46. Furuno T and Nakanishi M: Live cell imaging to study signaling molecules in allergic reactions. Biol Pharm Bull 28: 1551-1559, 2005.

47. De Benedetto A, Qualia CM, Baroody FM and Beck LA: Filaggrin expression in oral, nasal and esophageal mucosa. J Invest Dermatol 128: 1594-1597, 2008.

48. Van den Oord RAHM and Sheik A: Filaggrin gene defects and risk of developing allergic sensitization and allergic disorders: systematic review and meta-analysis. BMJ 339: b2433, 2009.

49. Hsu C-K, Akiyama M and Shimizu H: Filaggrin: an emerging star in atopic march. J Formos Med Assoc 107: 429-431, 2008.

50. Seguchi T, Cui CY, Kusuda S, Takahashi M, Aisu K and Tezuka T: Decreased expression of filaggrin in atopic skin. Arch Dermatol Res. 288: 442-446, 1996.

51. Sugiura H, Ebise H, Tanake K, et al: Large scale DNA microarray analysis of atopic skin lesions shows overexpression of epidermal differentiation gene cluster in the alterative pathway and lack of protective gene expression in the cornified envelop. Br J Dermatol 152: 146-149, 2005

52. Palmer CN, Ismail T, Lee SP, Terron-Kwiatkowski A, Zhao Y, Liao H, Smith FJ, McLean WH and Mukhopadhyay S: Filaggrin null mutations are associated with increased asthma severity in children and young adult. J Allergy Clin Immunol 120: 64-68, 2007.

53. Ying S, Meng Q, Corrigan CJ and Lee TH: Lack of filaggrin expression in the human bronchial mucosa. J Allergy Clin Immunol 118: 1386-1388, 2006.

54. Sandilands A, Sutherland C, Irvine AD and McLean WH: Filaggrin in the frontline: role in skin barrier function and disease. J Cell Sci 122: 1285-1294, 2009. 